Ann. Biol. anim. Bioch. Biophys., 1976, 16 (4), 603-622.

\title{
INFLUENCE DE L'INGESTION D'ACIDE CAPROÏQUE SUR L'UTILISATION DE L'ÉNERGIE ET DE L'AZOTE INGÉRÉS PAR LE RAT EN CROISSANCE EN FONCTION DE L'ÂGE DES ANIMAUX ET DE LA TENEUR EN AZOTE DES RÉGIMES
}

\author{
Marie-Claude CHENAT, B. AUROUSSEAU et M. VERMOREL \\ avec la collaboration technique de Françoise DuBorsset, \\ Simone Gasnet, Marinette Martinaud, G. Moins et R. Soucher \\ Laboratoire d'Étude du Métabolisme énergétique, \\ Centre de Recherches de Clermont-Ferrand, I. N.R. A., \\ Theix, Saint Genès Champanelle, 63110 Beaumont
}

\section{RÉSUMÉ}

De jeunes rats mâles de souche Wistar CF ont été utilisés pour déterminer les effets de l'ingestion de tricaproîne sur l'utilisation de l'énergie et de l'azote des rations d'animaux pris à deux stades physiologiques différents (Expérience I) ou recevant des régimes comportant des taux azotés différents (Expérience II).

L'expérience I a été réalisée selon le principe de la calorimétrie indirecte en chambres respiratoires de type circuit fermé, sur des rats recevant des régimes équilibrés au taux de $\mathrm{I}, 56 \mathrm{mg}$ d'azote digestible par Kjoule de EM ( $\mathrm{mg} \mathrm{Nd/KJ} \mathrm{EM).} \mathrm{Chez} \mathrm{les} \mathrm{rats} \mathrm{prépubères} \mathrm{(I} \mathrm{Io} \mathrm{g}$ de poids vif), par rapport aux animaux témoins, ceux recevant la tricaproïne ont présenté des besoins d'entretien pour la croissance (67 p. Ioo contre 7I p. Ioo) plus faibles. L'utilisation de l'énergie et de l'azote ingérés est améliorée, chez ces animaux, jusqu'à un certain niveau de consommation et diminué $a u$-delà. Chez les rats pubères ( $220 \mathrm{~g}$ de poids vif), les animaux recevant la tricaproïne ont présenté des rendements de EM pour la croissance très réduits (45 p. Ioo contre $82 \mathrm{p}$. 100) mais le faible niveau apparent du besoin d'entretien chez ces animaux conduit à penser à une liaison curvilinéaire entre EM et EF (Energie Fixée). Chez des animaux pris à ce stade physiologique, l'utilisation de l'azote ingéré est réduit jusqu'à un certain niveau de consommation et amélioré au-delà.

L'expérience II a été réalisée selon le principe d'abattage et analyse des carcasses et selon un schéma factoriel combinant 3 niveaux d'azote dans les régimes $(I, 20-1,36$ et $\mathrm{I}, 53 \mathrm{mg} \mathrm{Nd} / \mathrm{KJ}$ EM) et 3 niveaux de tricaproïne (o, ro ou $20 \mathrm{p}$. Ioo en énergie). L'utilisation de l'énergie et de l'azote ingérés ont été améliorées par l'introduction dans les régimes de tricaproïne, cet effet étant d'autant plus marqué que le régime est plus riche en azote. L'énergie fixée par les animaux expérimentaux a ainsi été accrue de 7 à 12 p. Ioo par rapport à celle fixée par les animaux témoins correspondants (même taux azoté des rations). De même, l'azote fixé a été accru de 2 à I I p. Ioo. La teneur des foies des animaux en énergie et en azote a été significativement accrue, ce qui souligne le rôle du foie chez les animaux recevant des acides gras à chaîne moyenne. Pour rendre compte des effets globaux observés, on a retenu l'hypothèse d'un effet insulino-sécréteur particulier de l'acide caproïque. 


\section{INTRODUCTION}

Le développement des tissus chez les animaux en croissance d'un type génétique donné est contrôlé par l'énergie qu'ils ingèrent. D'une part, globalement, par les quantités d'énergie ingérée (FULLER et al., I973, chez le Rat, WALKER et FAICHNEY, I964; ainsi que BLACK et GRIFFITHS, I975, chez l'Agneau) d'autre part plus spécifiquement, par la nature des sources d'énergie employées (SIEDLER et al., I962; Sinclair et Crawford, I973; Dror et al., I973, chez le Rat).

De nombreux travaux ont montré un effet favorable sur la croissance du Rat, des acides caproïque (MoHrhauer et Holman, I967). caprylique (Boli.inger et, REISER, 1956 ; HARKINS et SARRETT, I968) ou des mélanges d'acides gras à chaîne moyenne (KAUNITZ et al., I968). Mais ces travaux ne donnent aucune indication sur l'utilisation des nutriments ingérés ni sur les proportions de tissus formés.

Une première série d'études a permis de préciser l'influence de l'acide caprylique sur l'utilisation de l'énergie et de l'azote ingérés par le Rat en croissance : à énergie métabolisable (EM) ingérée égale, cet acide accroît la fixation d'azote chez le Rat prépubère (AUROUSSEAU et VERMOREI, I97I) et réduit la fixation d'énergie d'autant plus efficacement que l'animal est plus âgé et le niveau d'ingestion plus élevé (Aurousseau et al., I97I; Aurousseau, I972). Les deux expériences rapportées ci-dessous ont été entreprises en vue d'apporter des précisions du même ordre sur l'influence de 1'acide caproïque.

\section{MATÉRIEL ET MÉTHODES}

\section{A. - Animaux et schéma expérimental}

De jeunes rats mâles de souche Wistar CF, sevrés au poids moyen de $45 \mathrm{~g}$ ont reçu un aliment aggloméré du commerce pendant une période d'une semaine environ puis ont été adaptés aux différents régimes expérimentaux correspondant aux 2 expériences suivantes :

- Étude de l'influence de l'ingestion d'acide caproïque sur l'utilisation des nutriments, à deux stades physiologiques différents (expérience I).

- Étude de l'interaction entre la concentration en acide caproïque et en matières azotées du régime sur les quantités et les proportions des tissus formés (expérience II).

L'expérience I, réalisée par la méthode de calorimétrie indirecte, a porté sur la comparaison de l'utilisation de 2 régimes offerts à volonté à des rats prépubères pris à un poids moyen de $107 \pm 7 \mathrm{~g}$ (1o animaux expérimentaux et 9 animaux témoins) ou à des rats pubères pris à un poids moyen de $218 \pm 9 \mathrm{~g}$ ( 7 animaux expérimentaux et 8 animaux témoins). Les deux régimes, équilibrés au niveau de $1,53 \mathrm{mg}$ d'azote digestible par Kjoule d'énergie métabolisable $(1,53 \mathrm{mg}$ $\mathrm{Nd} / \mathrm{KJ} \mathrm{EM}$ ), comportaient o ou $20 \mathrm{p}$. Ioo d'énergie sous forme de tricaproïne (tabl. I).

L'expérience II a été réalisée selon un schéma factoriel, en utilisant la méthode des abattages comparés. Elle a porté sur 170 rats répartis en Io lots homologues en fonction du poids vif et du gain de poids vif au cours des 3 jours précédant la mise en lot. Un lot utilisé comme témoin a été abattu en début d'expérience et chacun des 9 autres lots (tabl. I) a reçu, pendant une période de $2 \mathrm{I}$ jours avant l'abattage, un régime équilibré aux niveaux de I,20-I,36 ou I,53 $\mathrm{mg} \mathrm{Nd/KJ} \mathrm{EM}$ et comportant o, Io ou 20 p. Ioo d'énergie sous forme de tricaproïne. Les distributions d'aliment ont été ajustées chaque jour en vue d'obtenir des ingestions de EM voisines pour chacun des 9 lots. 


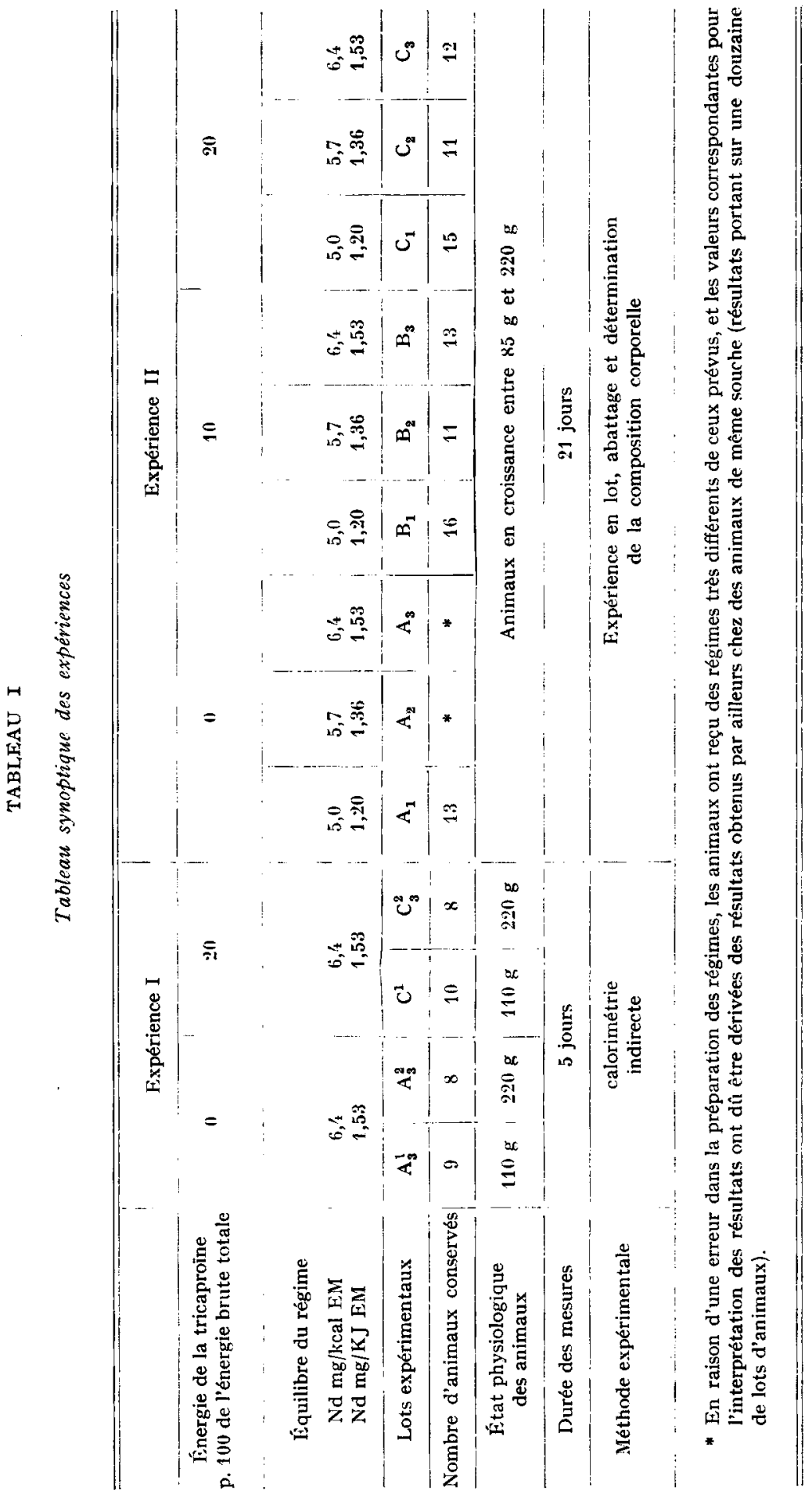




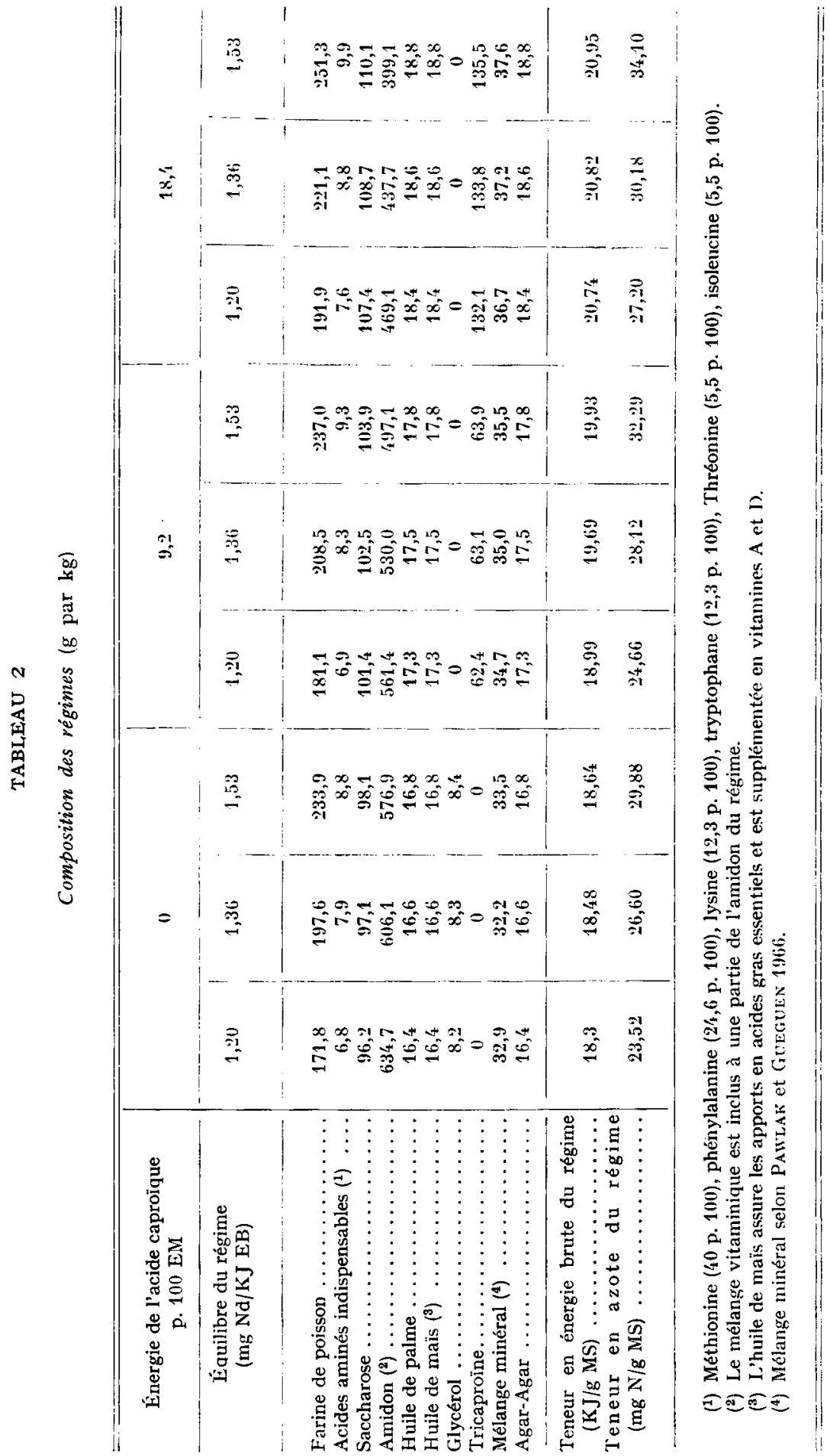




\section{B. - Régimes}

Les rats ont reçu des régimes semi-synthétiques, à base d'amidon et de farine de harengs de Norvège supplémentée en acides aminés indispensables selon les besoins du Rat en croissance définis par Pawlak et Pion (I968, 1971). Les régimes (tabl. 2) comportaient en outre 2 p. Ioo d'huile de maîs (assurant la couverture des besoins en acides gras essentiels), 5 p. Ioo d'huile de palme, $8 \mathrm{p}$. 1oo de saccharose et $3,5 \mathrm{p}$. Ioo de mélange minéral et vitaminique.

Les régimes ont été constitués selon un schéma factoriel combinant 3 niveaux d'azote ( $\mathrm{r}, 2 \mathrm{O}-$ $\mathrm{r}, 36$ ou $1,53 \mathrm{mg} \mathrm{Nd/KJ} \mathrm{EM)} \mathrm{à} 3$ taux de tricaproïne (o, ro ou $20 \mathrm{p}$. 100).

Pour chaque niveau azoté, les régimes expérimentaux ont été constitués à partir des régimes témoins correspondants de sorte que l'acide caprö̈que soit substitué à une partie de l'amidon sur la base d'apports égaux d'EM. L'acide caproïque a été introduit dans les régimes sous forme de tricaproïne : en compensation du glycérol introduit ainsi dans les régimes, des quantités équivalentes ont été introduites dans les régimes témoins correspondants.

\section{C. - Mesures et traitement des échantillons}

Les quantités d'aliments ingérés par les animaux ont été calculées quotidiennement par différence entre les quantités de matière sèche distribuées et refusées.

L'utilisation digestive et métabolique des aliments a été déterminée, après une période d'adaptation de 5 jours, au moins, sur des périodes de 5 jours, dans le cas de l'expérience $I$, en chambres respiratoires, ou de ro jours dans le cas de l'expérience II. Les techniques de récolte et de conservation des fèces et des urines ont été décrites par Vermorel et al. (1973). Les analyses ont été réalisées individuellement sur les urines et les fèces des animaux utilisés dans l'expérience I et sur l'ensemble des prélèvements effectués pour tous les animaux d'un même lot pour les mesures de digestibilité correspondant à l'expérience II. Pour cette expérience, les mesures ont été réalisées sur des groupes de 6 animaux recevant les régimes $A_{0}, B_{2}$ ou $C_{2}$ comportant respectivement o, 10 ou $20 \mathrm{p}$. Ioo d'énergie sous forme de tricaproïne et 1,02 ou 1,36 mg Nd/KJ EM. Le plan expérimental prévoyait d'effectuer les mesures de digestibilité sur le régime $\mathrm{A}_{2}$ équilibré au niveau de $1,36 \mathrm{mg} \mathrm{Nd} / \mathrm{KJ}$ EM, mais par suite d'une erreur de préparation, le régime obtenu a été $\mathrm{A}_{0}$. Pour l'expression des résultats, les pourcentages de EM dans l'énergie brute de chacun des régimes ont été obtenus à partir des valeurs déterminées en cage à métabolisme pour les régimes $\mathrm{A}_{0}, \mathrm{~B}_{2}$ et $\mathrm{C}_{2}$, par un calcul tenant compte du bilan d'azote obtenu par la méthode des abattages comparés.

Dans l'expérience I, les échanges respiratoires des rats ont été mesurés pendant 5 jours consécutifs à l'aide de 4 chambres respiratoires du type circuit fermé ; les bilans énergétiques (bilan QR et bilan $\mathrm{C}-\mathrm{N}$ ) et les bilans azotés ont été établis sur période globale de 5 jours, selon la méthode décrite précédemment (Vermorel et al., 1973). La production de chaleur des rats a été calculée à l'aide de la formule de Brouwer proposée par la FEZ (1964); cependant, dans le cas de l'utilisation de l'acide caproïque à des fins énergétiques, cette formule entraîne une erreur de $2,8 \mathrm{p}$. Ioo par excès; nous avons donc introduit une correction correspondant à cette observation en estimant la quantité d'acide caproïque catabolisée par les animaux.

Dans l'expérience II, lors de l'abattage, le tube digestif des rats a été vidé de son contenu et le foie a été prélevé. Les carcasses et les foies ont été conservés à une température de $-15^{\circ} \mathrm{C}$. Les carcasses des lots entiers de rats ont été broyées et analysées ensemble selon le schéma décrit antérieurement (AUROUSSEAU, 1972). Les dosages ont été effectués en triple sur 2 échantillons différents issus du premier broyage d'un même lot. Les résultats d'expériences antérieures réalisées sur des lots comparables de rats analysés individuellement ayant montré que le coefficient de variation à l'intérieur du lot, des teneurs en énergie, en azote et en lipides des carcasses est voisin de $6 \mathrm{p}$. Ioo, nous avons retenu cette valeur pour l'interprétation statistique des résultats obtenus sur des lots entiers.

Pour faciliter la mise en évidence des différences entre les lots, les bilans énergétiques et d'azote ont été corrigés pour les différences de EM ingérées et exprimées en valeur relative par rapport aux valeurs observées pour le régime témoin $A_{1}$, prises pour référence (valeur 100). On a également utilisé la notion d'efficacité de l'utilisation de l'énergie de l'acide caprö̈que comparée à celle de l'amidon (Aurousseau et al., I97I). Elle correspond au rapport de la quantité de EM d'amidon qui, apportée en substitution à l'acide caproïque des régimes expérimentaux, aurait permis une fixation d'énergie comparable, sur la quantité de EM d'acide caproïque reçue (tabl. 5 bis). 


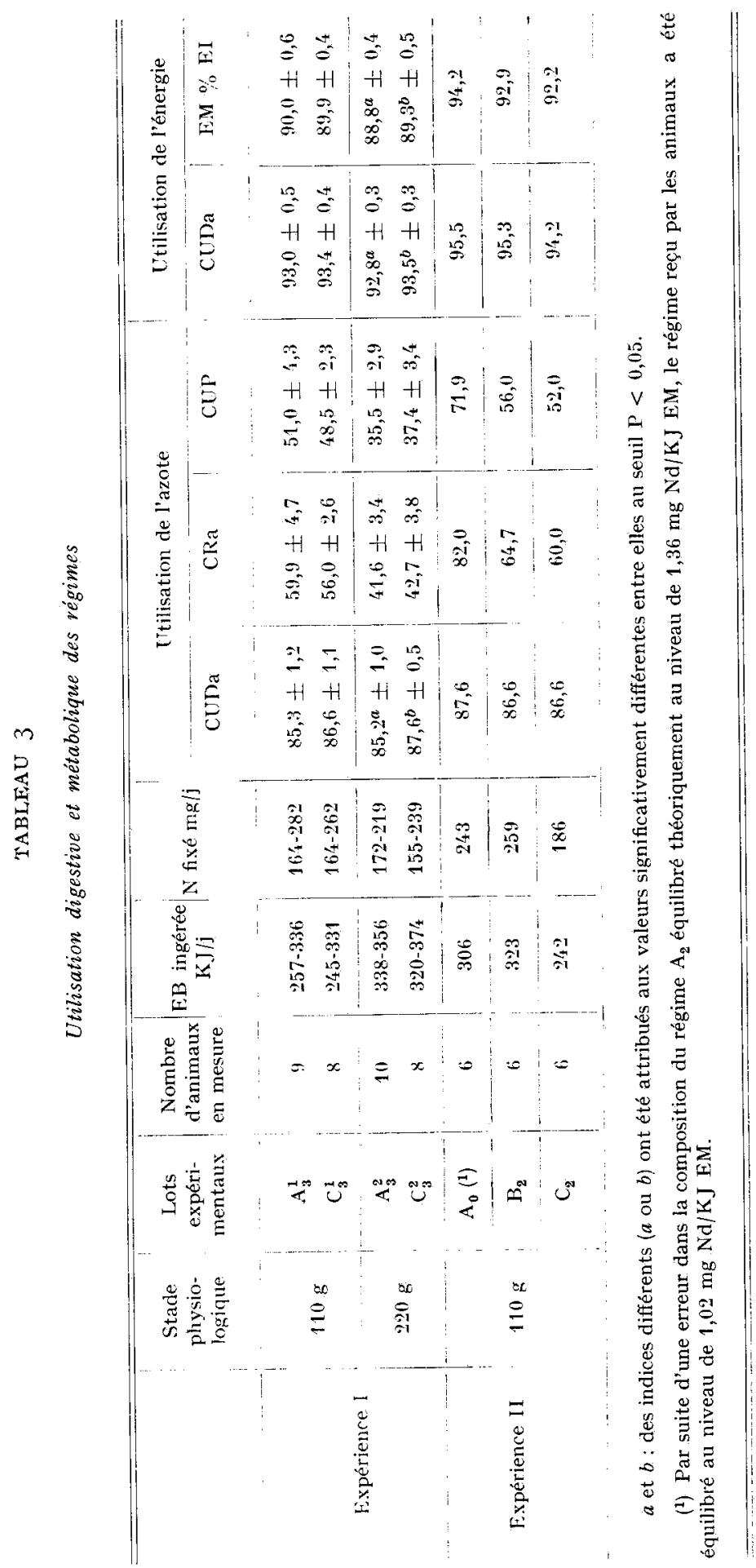




\section{RÉSULTATS}

\section{r. - Utilisation digestive des régimes}

Dans l'expérience I, les coefficients d'utilisation digestive de l'azote $(86,6$ contre 85,3 chez les animaux de $220 \mathrm{~g})$ et de l'énergie $(93,4$ contre 93,0 chez les animaux de IIo $\mathrm{g}$ et 93,5 contre 92,8 chez les animaux de $220 \mathrm{~g}$ ) ont été améliorés par la présence d'acide caproïque dans les régimes, cette amélioration étant significative dans le cas des animaux de $220 \mathrm{~g}$ (tabl. 3).

Dans l'expérience II, l'ingestion de tricaproïne a exercé dans l'ensemble un effet inverse, et a réduit de I point, en moyenne le coefficient d'utilisation digestive de l'azote, et de 0,2 à $I, 3$ points le coefficient d'utilisation digestive de l'énergie.

Dans ces conditions, sous l'effet de l'ingestion de tricaproïne, les proportions de EM dans les régimes ont été accrues dans l'expérience $I$ et réduites de $I$ point en moyenne dans l'expérience II. De plus, les apports d'acide caproïque aux niveaux de 9,2 et $\mathrm{I} 8,4 \mathrm{p}$. Ioo de l'énergie brute des régimes ont représenté ro et $20 \mathrm{p}$. Ioo environ de EM des régimes ( 9,9 à IO,I et I 9,8 à 20,2 p. I00, respectivement).

\section{2. - Quantités ingérées et vitesse de croissance}

Dans les 2 expériences, les quantités d'aliment ingérées ont été importantes et les gains de poids vif élevés (tab1. 4 et 5), ce qui traduit une appétibilité satisfaisante de la tricaproïne. Dans l'expérience I, chez les rats de IIo g, les animaux recevant de la tricaproine ont consommé en moyenne $5 \mathrm{p}$. Ioo de moins de EM que ceux du lot témoin et leur gain de poids vif moyen a été inférieur de $0,6 \mathrm{~g}$ (tabl. 4). Au cours de la période intermédiaire et de la deuxième série de mesures au poids de $220 \mathrm{~g}$, les quantités d'aliment distribuées aux rats du lot témoin ont été légèrement limitées pour que les quantités de FM ingérée soient comparables à celles des rats recevant de la tricaproïne. Les quantités moyennes d'EM ingérée sont de ce fait très voisines et la variabilité est très faible chez les rats témoins. Le gain de poids vif moyen des rats recevant de la tricaproïne a été légèrement inférieur mais non significativement différent de celui des rats témoins.

Dans l'expérience II, les quantités moyennes de EM ingérées ont été très voisines, en raison du contrôle des consommations et le coefficient de variabilité intra lot a été de $2 \mathrm{p}$. Ioo. Le gain de poids vif a varié de 5,8 à $6,5 \mathrm{~g}$ par jour, augmentant à la fois avec la teneur en acide caproïque et avec la teneur en matières azotées des régimes, mais seule la différence entre le lot $C_{3}$ et les lots $A_{1}$ et $B_{1}$ est significative.

\section{3. - Utilisation de EM}

Dans l'expérience I et chez les rats de Iro $\mathrm{g}$, la quantité d'énergie fixée par les animaux qui ont reçu de l'acide caproïque est légèrement supérieure (5 à 2 p. Ioo) à celle fixée par les animaux témoins mais la différence n'est pas significative en raison de la variabilité importante observée dans le lot expérimental. Les régressions entre l'énergie fixée et EM (fig. I et 2) mettent en évidence, chez les animaux ingérant 


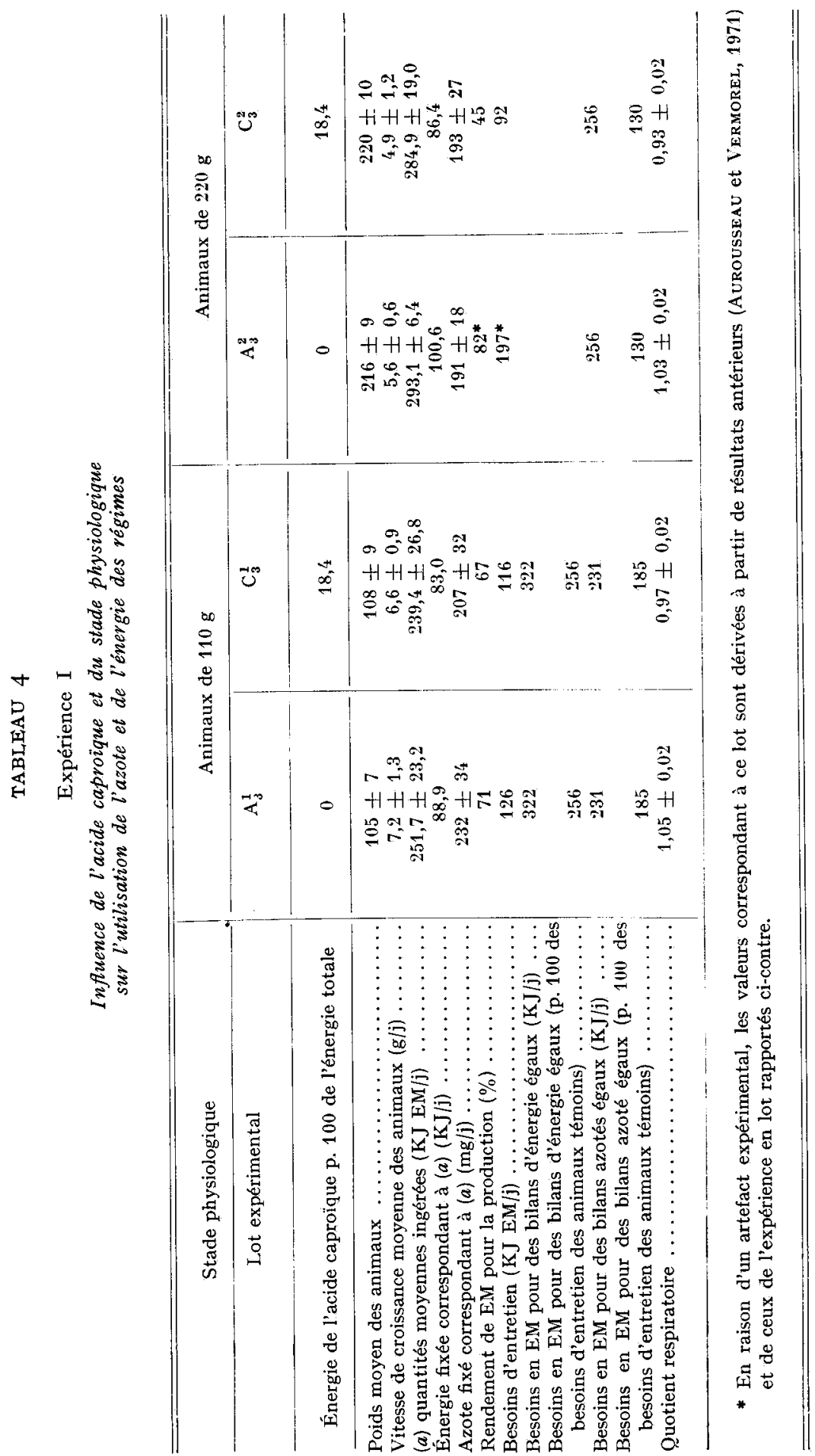


de la tricaproine, une légère réduction du rendement de EM pour la croissance $(67,3$ contre 70,6 p. I00) accompagnée d'une diminution du besoin d'entretien (II6 contre I26 KJ EM par jour) mais les différences ne sont pas significatives.

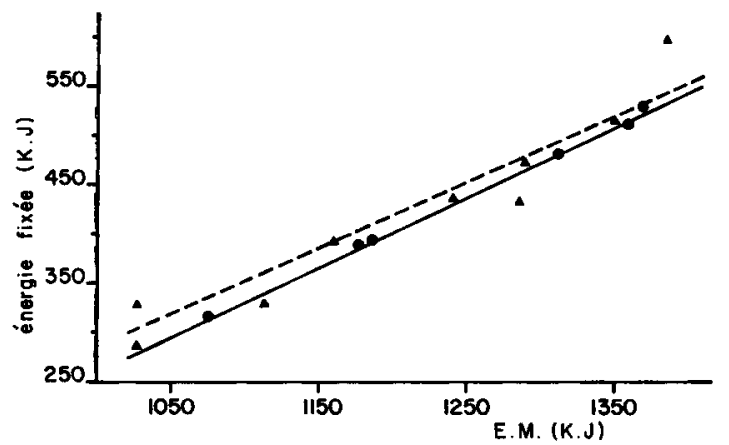

FIG. I. - Variations, en fonction de EM ingêrée,

$$
\begin{array}{ccc}
\text { des quantités d'énergie fixées en } 5 \text { jours chez des rats de } 110 \mathrm{~g} \\
\text { TRI C6 - } \mathrm{Y}=0,673 x-390,8 & r=0,94 \\
\text { Témoin }-\mathrm{Y}=0,706 x-443,3 & r=0,99
\end{array}
$$

Chez les rats de $220 \mathrm{~g}$, les quantités d'énergie fixée, pour une même ingestion de EM, sont plus élevées chez les rats témoins que chez les rats recevant de la tricaproïne. La faible variation des quantités de EM ingérée par les rats témoins ne permet pas de déterminer avec précision le rendement de EM pour la croissance.

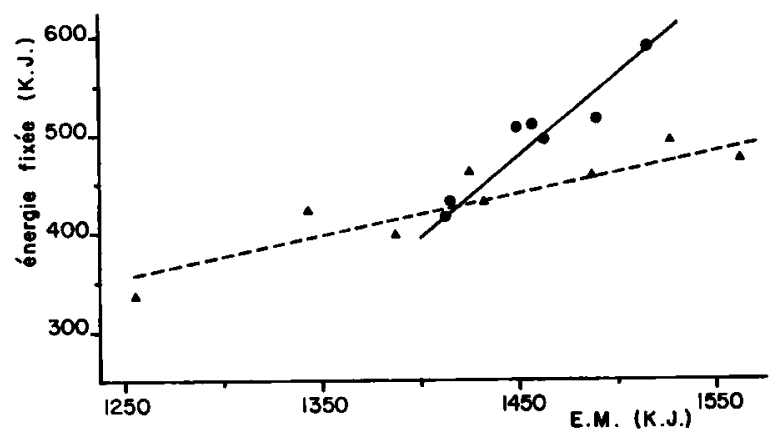

FIg. 2. - Variations, en fonction de EM ingérée,

des quantités d'énergie fixée en 5 jours, chez des rats de $220 \mathrm{~g}$

$$
\begin{array}{lll}
\text { TRI C6 } 4---- & \mathrm{Y}=0,447 x-204,6 & r=0,9 \mathrm{I} \\
\text { Témoin }-\mathrm{Y}=\mathrm{I}, 47 \mathrm{I} x-\mathrm{I} 65 \mathrm{I}, 4 & r=0,95
\end{array}
$$

Dans l'expérience II, une erreur dans la composition de 2 des régimes témoins $\left(A_{2}\right.$ et $\left.A_{3}\right)$ a conduit à des concentrations azotées trop élevée ou trop basse qui ont interdit une comparaison directe entre les lots témoins et les lots expérimentaux correspondants. Les résultats montrent (tab1. 5) que l'ingestion de tricaproïne a entraîné une amélioration de l'utilisation de EM des régimes ; cette dernière s'est traduite, à EM ingérée égale, par une augmentation de la quantité d'énergie fixée, de 2 à $6 \mathrm{p}$. Ioo par rapport au lot témoin recevant le régime le moins riche en azote. Cet effet augmente à la fois avec la teneur en tricaproïne et avec la concentration azotée du régime. 


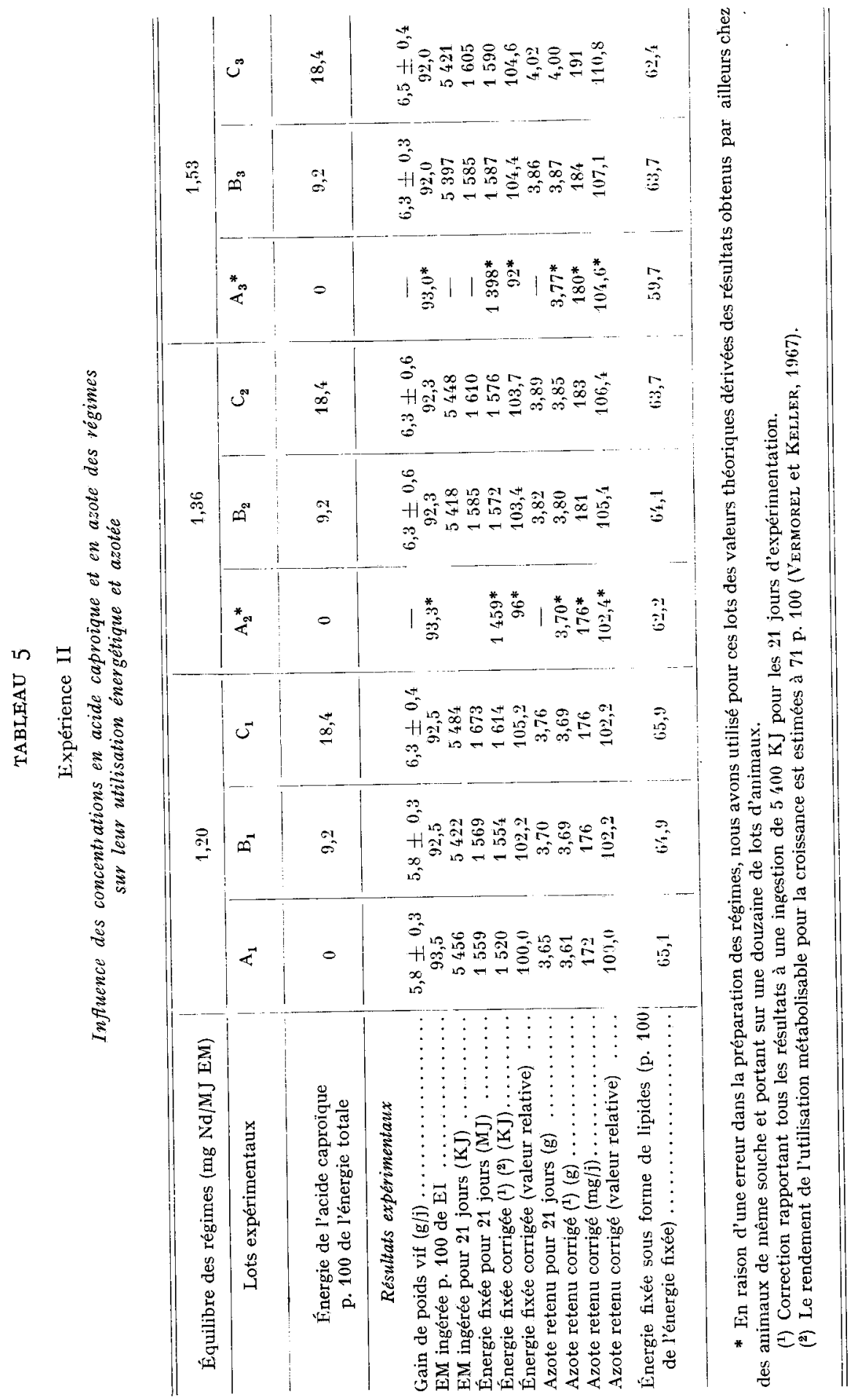




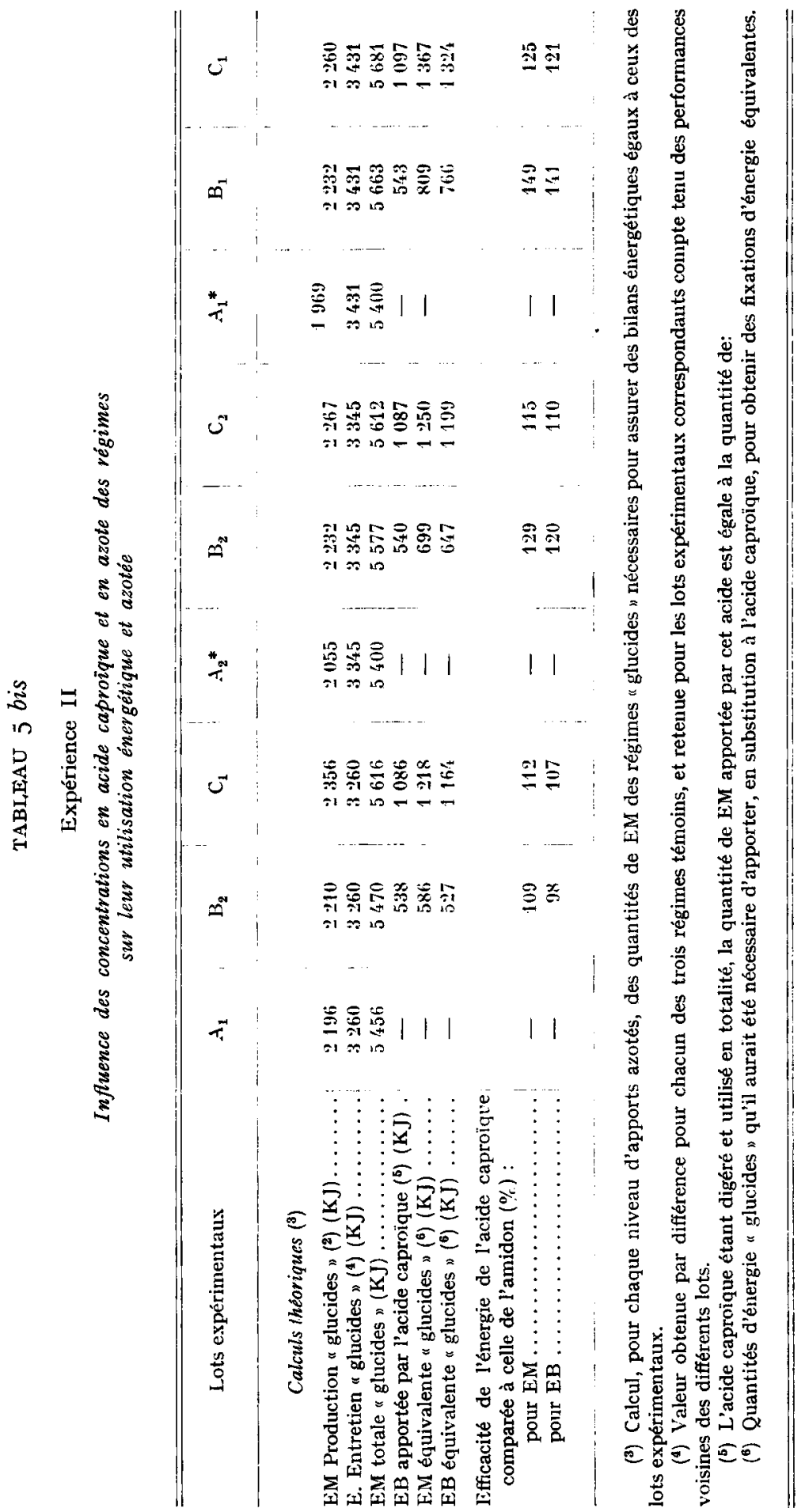




\section{4. - Utilisation métabolique de l'azote}

Les résultats obtenus dans l'expérience I montrent que l'ingestion de tricaproïne a modifié l'utilisation métabolique de l'azote de façon différente suivant le stade physiologique des rats. La régression entre l'énergie fixée sous forme de protéines et 1'EM ingérée (fig. 3) met en évidence que l'acide caproïque a, chez les rats

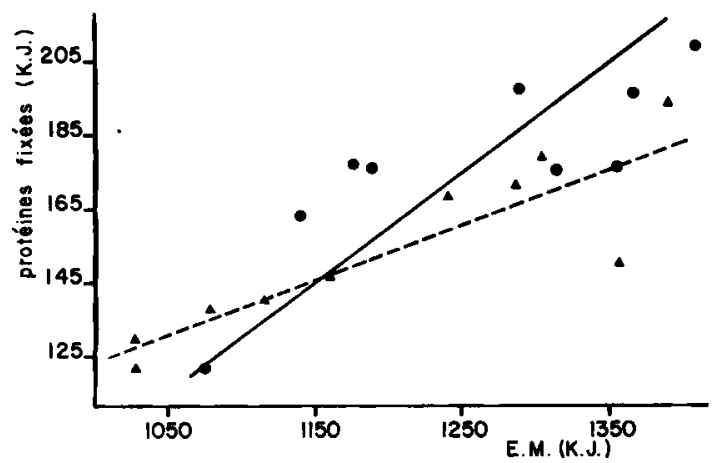

Fig. 3. - Variation, en fonction de EM ingêrée, des quantités d'ènergie fixées sous forme de protéines en 5 jours chez des rats de $110 \mathrm{~g}$

$$
\begin{array}{lll}
\text { TRI C6 } & \mathrm{Y}=0,153 x-28,9 & r=0,87 \\
\text { Témoin } & \mathrm{Y}=0,247 x-\mathrm{I} 37,6 & r=0,83
\end{array}
$$

de Iro g un effet dépressif, au-delà d'une consommation d'EM de $230 \mathrm{KJ}$ par jour, correspondant à un gain de poids vif de $6 \mathrm{~g}$ par jour. Chez les rats de $220 \mathrm{~g}$, en revanche, 1'acide caproïque tend à exercer un effet favorable au-delà d'un certain niveau de consommation, mais la faible variation des quantités de EM ingérée par les rats témoins ne permet pas d'analyser le phénomène avec précision (fig. 4).

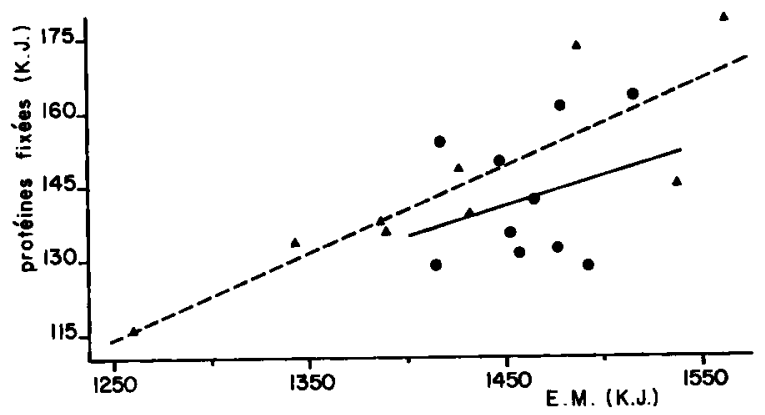

FIG. 4. - Variation, en fonction de EM ingérée, des quantités d'énergie fixées sous forme de proteines en 5 jours ches des rats de $220 \mathrm{~g}$

$\begin{array}{lll}\text { TRI C6 } & \mathrm{Y}=0,173 x-102,3 & r=0,82 \\ \text { Témoin }- & \mathrm{Y}=0,119 x-32,0 & r=0,28\end{array}$

Pour les consommations moyennes observées dans cette expérience à chaque stade physiologique, la diminution de la quantité d'azote fixé a été de $4 \mathrm{p}$. Ioo chez les rats de I Io g et l'augmentation de $7 \mathrm{p}$. Ioo chez les rats de $220 \mathrm{~g}$. Ces phéno- 
UTILISATION ÉNERGÉTIQUE DE L'ACIDE CAPROÏQUE

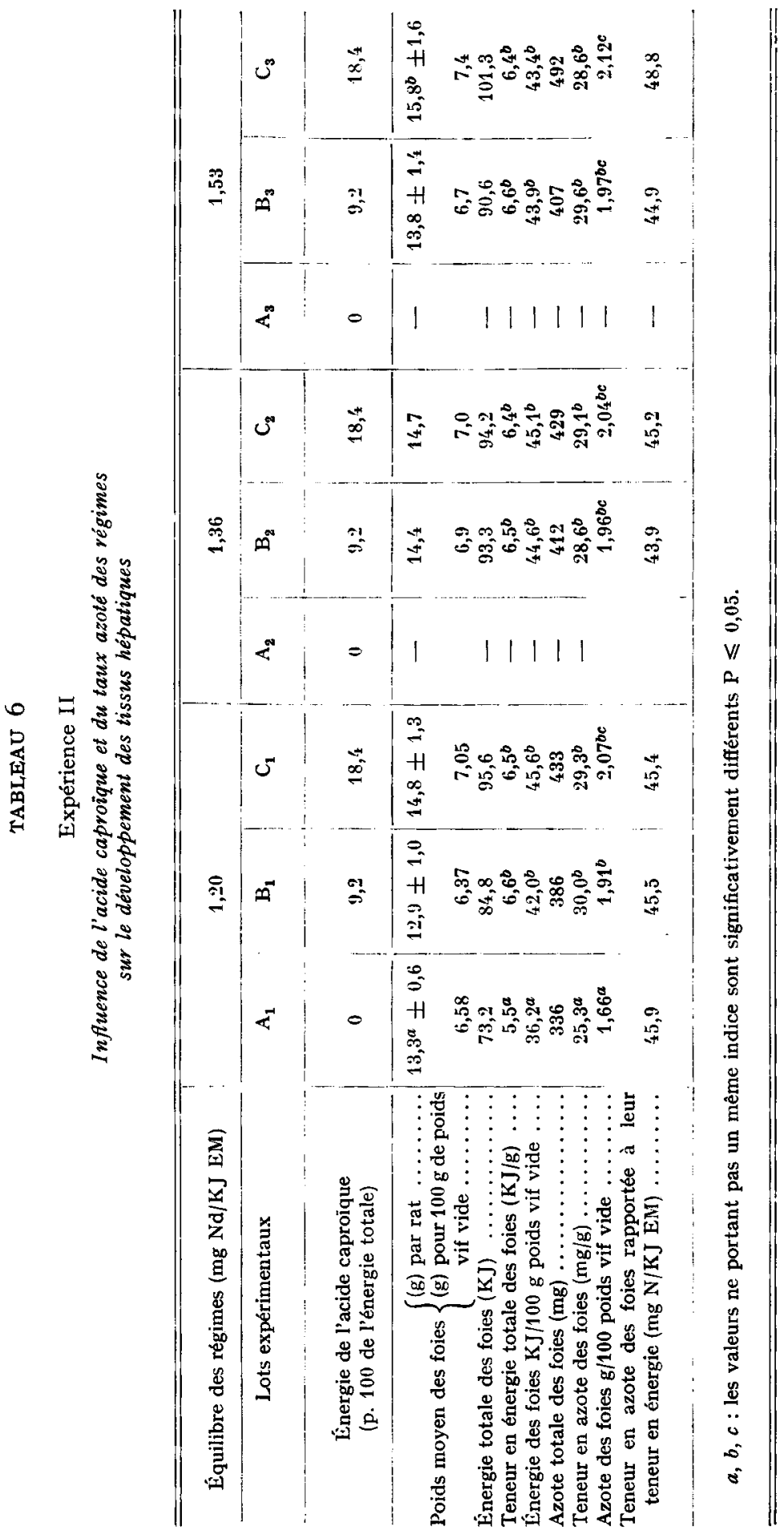


mènes se traduisent également par une diminution de 2,5 points à 3 points du CUP et du CR chez les rats de IIo g et une augmentation de 2 points du CUP et du CR moyens chez les rats de $220 \mathrm{~g}$. En raison du nombre réduit de données et de leur variabilité, aucune de ces différences n'est significative.

Dans l'expérience II, l'ingestion de tricaproïne $a$, dans tous les cas entraîné une amélioration de l'utilisation de l'azote du régime (tabl. 5) qui s'est traduite par une augmentation de 2 à II p. roo des quantités d'azote fixées. L'amplitude de cet effet a été accrue à la fois par l'introduction de proportions croissantes de tricaproïne dans le régime et par l'augmentation de la concentration azotée.

Chez les animaux qui ont reçu de la tricaproïne, l'état d'engraissement, défini par le pourcentage d'énergie fixée sous forme de lipides dans l'énergie fixée totale, est réduit par l'accroissement des proportions de matières azotées dans la ration, de façon importante dans le cas des régimes comportant ro $\mathrm{p}$. Ioo d'énergie sous forme de tricaproine (réduction de 3,5 points du régime $B_{3}$ équilibré au niveau de $1,53 \mathrm{mg} \mathrm{Nd} / \mathrm{KJ} \mathrm{EM}$ au régime $\mathrm{B}_{1}$ équilibré au niveau de $\mathrm{I,20} \mathrm{mg} \mathrm{Nd} / \mathrm{KJ} \mathrm{F} / \mathrm{M}$ ) et de façon moins marquée dans le cas des régimes comportant $20 \mathrm{p}$. Ioo d'énergie sous forme de tricaproïne (réduction de $I, 2$ points respectivement du régime $\mathrm{C}_{3}$ équilibré au niveau de $\mathrm{I}, 53 \mathrm{mg} \mathrm{Nd} / \mathrm{KJ} \mathrm{EM}$ au régime $\mathrm{C}_{1}$ équilibré au niveau de $\mathrm{I}, 20 \mathrm{mg} \mathrm{Nd} / \mathrm{KJ} \mathrm{EM}$ ).

\section{5. - Métabolisme des nutriments ingérés}

La mesure des consommations de $\mathrm{O}_{2}$ et des excrétions de $\mathrm{CO}_{2}$ des rats dans l'expérience I a permis de montrer (tabl. 4) que le quotient respiratoire moyen a été significativement réduit par l'ingestion de tricaproïne $(0,97$ contre $\mathbf{I}, 05$ chez les rats de $\mathrm{I}$ ro $\mathrm{g}$ et 0,93 contre $\mathrm{I}, 03 \mathrm{chez}$ les rats de $220 \mathrm{~g}$ ).

Par ailleurs, l'analyse des foies des animaux abattus en fin d'expérimentation lors de l'expérience II (tabl. 6), montre que l'ingestion d'acide caproïque a accru significativement le contenu total et les teneurs en énergie et en azote des foies des animaux correspondants ( $+\mathrm{I}_{3}$ à $+20 \mathrm{p}$. Ioo) ainsi que les proportions d'énergie et d'azote du foie par rapport au reste de la carcasse ( $+\mathrm{I}_{5}$ à +28 p. Ioo). Les différences observées portent essentiellement sur des modifications de composition plutôt que sur le développement de cet organe, qui n'est significativement augmenté que dans le cas du lot $\mathrm{C}_{3}$ (20 p. Ioo de tricaproïne et concentration en azote la plus élevée).

\section{DISCUSSION}

\section{I. - Utilisation des régimes et proportions de EM}

Les valeurs des CUD de l'azote des régimes témoins, observées dans ces 2 expériences, ont été en moyenne inférieures aux valeurs observées habituellement $(85,2$ à 87,6 p. Ioo contre 88,0 à 89,5 p. I00). Ces diminutions pourraient être dues à l'intervention d'un facteur défavorable dans l'alimentation (vieillissement et rancissement partiel de la farine de poisson par exemple). Dans ces conditions, et compte tenu des variations observées du CUD de l'azote à l'intérieur des lots témoins, et 
des effets contradictoires observés, il n'est pas possible de tirer de conclusions concernant l'influence de la tricaproïne sur l'utilisation digestive de l'azote des régimes.

De même, les valeurs des CUD de l'énergie ont été très variables $(92,8$ à $95,5 \mathrm{p}$. Ioo ainsi que celles des pourcentages de EM dans EB (88,8 à 94,2 p. Ioo) aussi bien pour les régimes témoins que pour les régimes comportant de la tricaproïne. Comme dans le cas de l'azote, les effets de l'acide caproïque ont été contradictoires et on ne peut tirer de conclusion.

Pour l'expérience en lot, nous avons calculé les proportions de EM dans EB pour chacun des 9 régimes à partir des valeurs déterminées pour les régimes $A_{0}$, $\mathrm{B}_{2}$ et $\mathrm{C}_{2}$ en cage à métabolisme. Nous avons tenu compte pour ce calcul des différences d'excrétion d'azote dans les urines déterminées en cage à métabolismes ou appréciées pour chacun des 9 lots à partir du bilan carcasse.

Les valeurs des proportions de EM dans EB attribuées aux différents régimes comportant 9,2 ou I8,4 p. Ioo de EB sous forme d'acide caproïque ont ainsi été les mêmes pour chaque niveau de concentration en azote des régimes (tabl. 5). Ce mode de calcul ne permet pas de tenir compte des variations de digestibilité de l'azote ingéré ni des variations d'excrétion urinaire de composés autre que l'urée selon le taux azoté des différents régimes, mais l'amplitude de l'erreur ainsi admise reste vraisemblablement très faible.

\section{2. - Utilisation de l'énergie métabolisable}

Dans l'expérience I, nous avons retenu, pour exprimer les résultats obtenus en chambres respiratoires, les valeurs du bilan $Q R$. En effet, bien que les deux bilans soient voisins, le bilan $\mathrm{CN}$ est en général plus élevé $(+2 \mathrm{a}+3 \mathrm{p}$. roo) en raison vraisemblablement de la sous-estimation du carbone urinaire. Par ailleurs, les consommations des animaux témoins ont été légèrement réduites au-delà du poids moyen de r Io g. Cette limitation des quantités ingérées peut avoir eu des effets durables sur le métabolisme des animaux et, en réduisant leurs besoins énergétiques (MAheindra et Milier, I969; Aurousseau, 1972) être à l'origine de la meilleure utilisation de EM par les rats témoins de $220 \mathrm{~g}$.

Les travaux sur l'utilisation de l'acide caproïque par le Rat en croissance rapportés ci-contre ont été réalisés à la suite d'expériences similaires relatives à l'étude de l'utilisation d'acide caprylique (AURousseau et al., I970; AUrousseau et VERMOREL, I97I ; AUROUSSEAU, I972). Il nous est apparu intéressant de comparer les effets de ces deux acides.

Le rendement de EM pour la croissance dans le cas de régimes comportant de 1'acide caprylique ou de l'acide caproïque varie de façon comparable avec l'âge des animaux mais diffère dans son amplitude. Fin effet, le rendement est non significativement accru par l'acide caprylique chez les animaux de $80 \mathrm{~g}$ (64 p. Ioo contre 62 p. Ioo pour un régime témoin), il est réduit sensiblement chez les animaux de IIo $\mathrm{g}$ recevant de 1 'acide caproïque $(67,3 \mathrm{p}$. Ioo contre $70,6 \mathrm{p}$. Ioo pour un régime témoin). Enfin, chez les animaux de $220 \mathrm{~g}$, il est beaucoup moins affecté par la présence d'acide caprylique que par celle d'acide caproïque (66 p. Ioo contre $45 \mathrm{p}$. Ioo alors qu'il est de 82 p. Ioo dans le cas de régimes témoins).

L'extrapolation des droites de régression linéaire reliant 1'énergie fixée à 1'EM ingérée permet un calcul théorique approché des besoins d'entretien des animaux : 
ces besoins, inchangés chez les animaux de $80 \mathrm{~g}$ ingérant de l'acide caprylique seraient, selon cette méthode de calcul, abaissés de $8 \mathrm{p}$. Ioo chez les animaux de I Io g ingérant de l'acide caproïque et de 14 p. Ioo et de 47 p. Ioo, respectivement, chez les animaux de $220 \mathrm{~g}$ ingérant de l'acide caprylique ou de l'acide caproïque. L'amplitude de ces variations théoriques rend très improbable leur validité et on peut penser que dans le cas de l'ingestion d'acides gras à chaîne moyenne, la régression reliant l'énergie fixée à l'EM ingérée prend une forme curvilinéaire.

Dans le cas de 1'expérience II, pour remplacer les résuitats manquants (lots $A_{2}$ et $A_{3}$ équilibrés aux niveaux respectifs de $I, 36 \mathrm{mg} \mathrm{Nd} / \mathrm{Kj} \mathrm{EM}$ ), nous avons estimé les quantités d'énergie et d'azote qu'auraient dû fixer les lots $A_{2}$ et $A_{3}$ à partir de données obtenues au laboratoire sur des lots de rats comparables recevant des régimes témoins de concentrations azotées variables; les résultats obtenus pour les 2 lots recevant les régimes de concentrations azotées trop élevée ou trop basse de la présente expérience ont été inclus dans ces données, et les chiffres obtenus correspondent bien aux données habituelles (TANAKa et al., I974).

Nous avons pu calculer ainsi (tabl. 5 bis) l'efficacité de l'utilisation de l'EM ou de 1'EB de l'acide caproïque comparée à celle de l'amidon. Elles subissent des variations importantes et prennent les valeurs extrêmes respectives de rog et I49 p. Ioo ou de 98 et I4I p. Ioo. Compte tenu de la méthode d'appréciation des proportions de EM dans chaque régime (cf. discussion ci-dessus, $\S$ I) il est possible que les valeurs de l'efficacité de EM de l'acide caproïque soient surestimées. Dans le cas de l'ingestion d'acide caprylique, l'efficacité de EM et de EB de cet acide variait respectivement dans les mêmes conditions de 76 à 87 p. roo (Aurousseau et al., i97o; Aurousseau, I972) et de 86 à II5 p. Ioo. Ainsi, l'acide caproïque qui réduit davantage, dans la plage de consommation observée, le rendement de l'utilisation de EM pour la croissance, que l'acide caprylique, conduit à une meilleure utilisation de l'énergie ingérée (EB ou EM) pour l'ensemble des fonctions d'entretien et de croissance des animaux.

\section{3. - Utilisation de l'azote ingéré}

La quantité d'azote fixé est reliée de façon linéaire aux quantités totales d'énergie et d'azote ingérés et au poids métabolique à la fois (BLACK et GRIFriTHS, I975). Dans ces conditions, on ne peut extrapoler à bilan d'azote nul les régressions calculées à partir des résultats de l'expérience I et liant les quantités d'azote fixé (exprimées en énergie) aux quantités de EM ingérées seules. L'allure générale de ces courbes semble, cependant, indiquer, qu'en présence d'acide caproïque les besoins en énergie pour assurer des bilans azotés nuls sont réduits chez les animaux de I Io g, et accrus chez les animaux de $220 \mathrm{~g}$. L'allure générale du phénomène était semblable dans le cas des animaux ingérant des régimes comportant de l'acide caprylique (AUROUSSEAU et Vermorel, I97 $\mathrm{x}$ ).

Les résultats de l'expérience II, complétés selon le calcul décrit au paragraphe précédent, font apparaître une interaction très nette entre les effets de l'acide caproïque et le taux azoté des régimes. L'azote ingéré est ainsi d'autant plus efficacement utilisé que le régime est plus riche à la fois en acide caproïque et en matières azotées. Cependant, compte tenu de ses effets sur l'utilisation de l'énergie ingérée, l'acide caproïque accroît non significativement l'état d'engraissement des animaux, cet effet étant d'autant plus net que le régime est plus riche en azote (tabl. 5). Ces 
interactions avec le taux d'azote dans les régimes n'avaient pas été observées dans le cas de l'acide caprylique.

L'effet insulino-sécréteur des acides gras à chaîne moyenne (SunYER et al., I969), apparemment maximum dans le cas de l'acide caproïque (Амво et al., I973) pourrait être à l'origine des effets observés.

\section{4. - Métabolisme des nutriments}

Compte tenu du fait, que les acides gras à chaîne moyenne sont facilement catabolisés en $\mathrm{CO}_{2}$ dans les tissus animaux in vitro (GREENBERGER et al., I965; SCHEIG et al., Ig68) aussi bien qu'in vivo (KIRSCHNER et al., I96I ; BolLINGER, I963 ; GREENBERGER et SkILMAN, I969), il nous est apparu intéressant d'étudier la signification du $Q R$ des animaux expérimentaux.

Dans un premier temps, nous avons calculé ainsi le $Q R$ théorique d'animaux catabolisant en $\mathrm{CO}_{2}$ la totalité de l'acide caproïque ingéré en remplacement d'une quantité isoénergétique de glucides, toutes choses égales par ailleurs (sans changement des quantités d'azote et d'énergie fixées). Dans ces conditions, le $Q R$ des animaux de 1 Io $\mathrm{g}$ et de $220 \mathrm{~g}$ serait abaissé de $\mathrm{I}, 05$ et $\mathrm{I}, 03$ respectivement, à 0,97 et 0,96 .

Dans un deuxième temps, nous avons poursuivi le calcul pour tenir compte des différences d'énergie et d'azote fixés, selon le niveau d'ingestion. Pour expliquer l'abaissement du $Q R$ par rapport aux valeurs théoriques on doit faire appel, dans la zone où l'acide caproïque améliore l'utilisation de l'énergie ingérée à l'intervention de synthèses de lipides à partir de glucides soustraits à la catabolisation en $\mathrm{CO}_{2}$. En effet, dans 1'hypothèse inverse où les synthèses de lipides supplémentaires s'effectueraient à partir d'acide caproique, le QR des animaux serait augmenté. Selon ce mode de raisonnement, l'acide caproïque serait ainsi en quasi totalité catabolisé en $\mathrm{CO}_{2}$.

Dans le cas d'animaux ingérant de la tricapryline (AUROUSSEAU et VERMOREL, 197I) les QR observés étaient également inférieurs à ceux des rats témoins (0,94 et 0,93 contre I,O5 et I,OI respectivement pour des animaux de $80 \mathrm{~g}$ ou de $220 \mathrm{~g}$ ). Mais dans ce cas, un calcul analogue à celui que nous avons fait ci-dessus pour l'acide caproique montre que le $Q R$ théorique d'animaux expérimentaux catabolisant en tơtalité l'acide caprylique ingéré est inférieur au $Q R$ observé. Pour expliquer ce phénomène, on peut émettre 1'hypothèse suivante : globalement, des lipides seraient synthétisés à partir de l'acide caprylique ingéré et, une partie des glucides utilisés, dans le cas d'animaux témoins, pour la lipogenèse seraient catabolisés en $\mathrm{CO}_{2}$. Cette hypothèse concorde avec des mesures de catabolisme d'acide caprylique marqué au carbone $\mathrm{I}_{4}$ rapportées antérieurement (AUROUSSEAU et al., I97I) et on retrouve une différence entre l'utilisation métabolique des deux acides.

Au niveau hépatique, l'accroissement de la teneur globale ou relative des foies, en énergie et en azote témoigne du rôle accru de cet organe chez les animaux ingérant de l'acide caproïque. Des observations similaires avaient pu être faites dans le cas d'animaux recevant de l'acide caprylique (Aurousseau et al., I970). Par ailleurs, de nombreux travaux (HAShim et al., I964; SchwaBe et al., I964; Bezard et al., I966 ; Bezard, Monneret et Boquillon, I966 ; Green, Berger 
et Skiliman, I969) montrent que le foie capte activement et en quasi totalité, les acides gras à chaîne moyenne absorbés par la voie porte.

L'accroissement de la teneur des foies en lipides est à rapprocher d'observations montrant que les acides gras à chaînes moyennes accroissent les synthèses d'acides gras longs à partir d'unités acétate (BRADY et GURIN, I950 ; REISER et al., I963) ainsi que l'émission par le foie de lipoprotéines (HASHim et al., I960; UzAwa et al., I964).

\title{
CONCLUSION
}

A la suite de ce premier travail, on peut dégager des résultats obtenus les aspects suivants :

- l'acide caproïque a amélioré à la fois l'utilisation de l'énergie et de l'azote ingérés, l'effet étant d'autant plus marqué que le régime était plus riche en azote ou en acide caproïque ;

- l'acide caproïque a amélioré l'utilisation de l'énergie ingérée dans certaines limites du niveau d'ingestion (jusqu'à un niveau de $256 \mathrm{p}$. roo ou de $156 \mathrm{p}$. roo des besoins d'entretien des animaux témoins respectivement pour les animaux de I ro $\mathrm{g}$ ou de $220 \mathrm{~g}$ ).

En ce qui concerne l'utilisation de l'azote ingéré, les effets de l'acide caproïque ont été différents selon l'âge physiologique des animaux :

- chez le jeune Rat de IIo g, l'acide caproïque a amélioré l'utilisation de l'azote ingéré dans la zone des bas niveaux d'ingestion (inférieurs à I85 p. I00 des besoins d'entretien des animaux témoins) ;

- chez les animaux de $220 \mathrm{~g}$, 1'acide caproïque a amélioré l'utilisation de l'azote ingéré dans la zone des hauts niveaux d'ingestion (supérieurs à I30 p. IOO des besoins d'entretien des animaux témoins).

Nous avons comparé ces résultats à ceux obtenus antérieurement avec l'acide caprylique et avancé des hypothèses sur les mécanismes responsables des différences observées au niveau des aspects globaux. Les deux acides semblent agir sur les mêmes voies métaboliques, mais induire des modifications d'intensités relatives variables des voies métaboliques concernées. Nous avons ainsi retenu l'hypothèse de travail selon laquelle l'acide caproïque pourrait, en particulier, accroître plus efficacement la sécrétion d'insuline.

Reçu pour publication en août 1975.

\section{SUMMARY}

\author{
EFFECT OF INTAKE OF CAPROIC ACID ON ENERGY \\ AND NITROGEN UTILIZATION IN GROWING RAT AS RELATED \\ TO AGE OF ANIMAL AND NITROGEN LEVEL IN DIET
}

Yong male rats of the Wistar CF strain are used to determine the effects of tricaproine intake on energy and nitrogen utilization in diets of animals at two different physiological stages (Experiment $\mathrm{I}$ ) or when given in rations containing various nitrogen levels (Experiment II). 
Experiment $I$ is carried out in closed-circuit respiration chambers using indirect calorimetry on rats given balanced diets containing $1.56 \mathrm{mg}$ digestible nitrogen per metabolizable energy (EM) Kjoule (mg Nd/KJ EM). Prepuberal rats (I Io g liveweight) receiving tricaproine have lower growth maintenance requirements than control animals (67 p. roo as against $7 \mathrm{I}$ p. Ioo). Utilization of energy and nitrogen is improved in these animals up to a certain consumption level and then decreases. Puberal rats $(220 \mathrm{~g}$ liveweight) given tricaproine present very reduced EM growth yields (45 p. 10o as against $82 \mathrm{p}$. 10o), but the apparent low level maintenance requirement leads us to believe that there is a curvilinear relationship between EM and EF (fixed energy). In animals taken at this physiological stage, the use of ingested nitrogen is diminished to a certain consumption level and then improves.

Experiment II is done by sacrificing the animals and analyzing the carcasses, following a factorial plan combining 3 nitrogen levels in the diets (I.20-I. 36 and r.53 mg Nd/KJ EM) and 3 tricaproine levels (o, Io or 20 p. Ioo in energy). Energy and ingested nitrogen utilization are improved by introducing tricaproine into the diets, the effect being more evident as the diet is richer in nitrogen. Energy fixed by experimental animals thus increases from 7 to 12 p. Ioo as compared to that fixed by the corresponding control animals (same nitrogen level in diets). Also fixed nitrogen is increased from 2 to I $_{\mathrm{p}} \mathrm{p}$. roo. The amount of energy and nitrogen are significantly increased in rat livers, thus emphasizing the role of the liver in subiects given medium chain fatty acids. The overall effects observed lead us to retain the hypothesis that caproic acid has a particular insulino-secretor effect.

\section{RÉFÉRENCES BIBLIOGRAPHIQUES}

Ambo K., Таканashi M., Tsuna T., 1973. Effect of feeding and infusion of short Chains fatty acids on glucose levels in sheep. $J$. Agric. Res., 24, 54-62.

Ash R. W., Pennington R. J., Reid R. S., I959. The effect of short chain fatty acids on blood glucose in sheep. Biochem. $J$, , $71,9 \mathrm{P}$.

Aurousseau B., De Groot L., Vermorel M., 1970. Étude comparée de l'utilisation énergétique de régimes riches en acide caprylique ou en acides gras insaturés. Ann. Biol. anim. Bioch. Biophys., 10, 703-706.

Aurousseau B., De Groot L., Bouvier J.C., Vermorel M., I97r. Utilisation métabolique des acides gras courts, moyens et longs par le Rat en croissance. In Energy metabolism of farm animals.

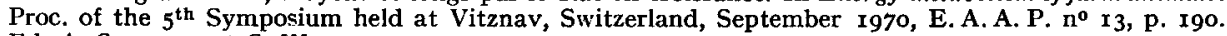
Ed. A. Schurch et C. Wenk.

Aurousseau B., Vermorel M., I97r. Caprylic acid utilization by growing rats at two different physiological states. Nutr. Rep. Intern., 4, 95-I02.

Aurousseat B., I972. Utilisation énergétique des acides caprylique, laurique et myristique par le Rat en croissance. Influence des restrictions alimentaires, Ann.Biol. Anim. Bioch. Biophys., 12, 263-280.

Bach A., Bieth N., Metais P., r973. Octanoate metabolism in the isolated perfused rat liver. I. Methodology and preliminary results. Arch. Sci. Physiol., 27, 55-65.

Bezard J., Clement G., Klepping J., Briet S., I966. Étude de la captation par le foie des acides gras à chaine courte et moyenne chez le Chien. Arch. Sci. Physiol., 20, 169-180.

Bezard J., Monneret-Boquillon M., I966. Captation par le foie en perfusion des acides gras à chaînes courtes et moyennes. I. Acides gras perfusés sous forme libre. Arch. Sci. Physiol., 20, 359-371.

Black J. L., Griffiths D. A., 1975. Effects of live weight and energy intake on the nitrogen metabolism and total nitrogen requirement of lambs. Brit. J. Nutr., 38, 399-413.

Bollinger J. N., 1963. The metabolism of fatty acids derived from dietary triglyceride. Ph. D. Thesis, Texas.

Bradr R. O., Gurin S., I950. The biosynthesis of radioactive fatty acids and cholesterol. J. Biol. Chem., 186, 46r-469.

Dror Y., Sassoon H. F., Watson J. J., Mack D. O., Johnson B. C., r973. Fat versus sucrose as the nonprotein calorie portion of the diet of rats. $J$. Nutr., 108, 342-346.

Fuller M. F., Boyne A. W., Atkinson T., Smart R., r973. The effect of increasing energy intake on the utilization of dietary protein of various qualities. Nutr. Rep. Int., 7, I75-I80.

McGarRY J. D., Foster D. W., I I97I. The regulation of ketogenesis from octanoic acid. The role of the tricarboxylic acid cycle and fatty acid synthesis. J. Biol. Chem., 246, I149-1159.

Greenberger N. J., Franks J. J., Isselbacher K. J., I965. Metabolism of I ${ }^{14} \mathrm{C}$ octanoîc and ${ }^{14} \mathrm{C}$ palmitic acid by the rat intestinal slices. Proc. Soc. Exp. Biol. Med., 120, 468-472. 
Greenberger N. J., Skilman T. G., r969. Medium chain triglycerides : physiological considerations and chemical implications. New Engl. J. Med., 280, 1045-1058.

Hashim S. A., Artega A., Van Itallie T. B., I960. Effect of a saturated medium chain triglyceride on serum lipids in man. Lancet, 1, 1105-I108.

Hashim S. A., Bergen S. S., Kreld K., Van Itallie T. B., I964. Intestinal absorption and mode of transport in portal vein of medium chain fatty acids. J. Clin. Invest., 43, I 238-1240.

INGEBRETSEN W. R. Jr., WAGLE S. R., I974. Studies on the effects of tricaprylin on gluconeogenesis and ketogenesis in isolated perfused liver. Proc. Soc. Exp. Biol. Med., 147, 578-580.

Kirshner J., Harris R.S., Ig6r. The effects of chain length on the metabolism of saturated fatty acids by the rat. J. Nutr., 73, 397-402.

Linscheer W. G., Slone D., Mitchell M. L., ig65. Octanoïc acid and carbohydrate metabolism. Clin. Res., 18, 327-330.

Mahendra C. C., Miller D. S., I969. The effect of depletion repletion on caloric utilization. Proc. Nutr. Soc., 28, 72A.

Sunyer F. X., Hashim S. A., Van Itallie T. B., I969. Insulin and ketone responses to ingestion of medium and long chain triglycerides in man. Diabetes, 181, 96-100.

Reiser R., Williams M. C., Sorrels M. S., 1963. Biosynthesis of fatty acids and cholesterol as related to diet fat. Arch. Biochem. Biophys., 102, 276-285.

Scheele C. W., Jansen B. P. 197r. Standardization of nitrogen balance experiments with rats. 2. Experimental procedure and techniques used in the evaluation of the experiments. ZTTF, 28, 24-28.

Scheig R., KLATSKin G., r968. Hepatic metabolism of ${ }^{{ }^{14}} \mathrm{C}$ octanoic and ${ }^{-14} \mathrm{C}$ palmitic acids. $J$. Am. Oil. Chem. Soc., 45, 3I-33.

Schwabe A. D., Bennet L. R., Browman L. P., 1964. Octanoíc acid absorption and oxydation in humans, J. Appl. Physiol., 19, 335-337.

Siedier A. J., Rice M. S., Maloney P. A., Lushbough C. H., Schweigert B. S., Ig62. The influence of varying levels of dietary protein carbohydrate and fats in the nutrition of the rat. $J$. Nutr., 77, $149-154$.

Sinclair A. J., Crawford M. A., r973. The effect of a low. fat maternal diet on neonatal rats. Brit. J. Nutr., 29, I27-I37.

TaNAKa H., Yamaguchi M., Kametaka M., I974. Body composition and utilization of protein and energy in growing rats at different dietary protein to energy ratios purified whole egg protein. $A g r$. Biol. Chem., 38, II13-1120.

Uzawa H., Schlierf G., Chirman S., Michaels G., Wood P., Kinsell L. W., ig64. Hyperglyceridemia resulting from intake of medium chain triglycerides. Am. J. Clin. Nutr., 15, 365-369.

Vermorel M., Keller J., 1967. Utilisation énergétique par le Rat en croissance, des principales céréales composant des régimes isoazotés et équilibrés en acides aminés. Ann. Zootech., 16, 223-234.

WALKER D. M., FaichneY G. J., 1964. Nitrogen balance studies with the milk fed lambs. 3. Effect of different nitrogen intakes on growth and nitrogen balance. Brit. J. Nutr., 18, 295-306.

Wiley J. H., Leveille G. A., 1973. Metabolic consequences of dietary medium chain triglycerides in the rat. $J$. Nutr., 108, 829-835. 